\title{
Post-Partum Hemorrhage is Still Killing Mothers in Bangladesh: An Observation from the 2016 Bangladesh Maternal Mortality Survey (BMMS)
}

Aminur Rahman ( $\sim$ draminur@icddrb.org )

International Centre for Diarrhoeal Disease Research https://orcid.org/0000-0003-1434-3883

Anne Austin

JSI Research and Training Institute Inc

Tahmina Begum

International Centre for Diarrhoeal Disease Research Bangladesh

lqbal Anwar

International Centre for Diarrhoeal Disease Research Bangladesh

\section{Short report}

Keywords: Postpartum Hemorrhage, Maternal mortality, Bangladesh, Oxytocin

Posted Date: August 10th, 2020

DOI: https://doi.org/10.21203/rs.3.rs-48884/v1

License: (a) (i) This work is licensed under a Creative Commons Attribution 4.0 International License.

Read Full License 


\section{Abstract}

The main cause of maternal death in Bangladesh is postpartum hemorrhage (PPH). PPH accounts for $31 \%$ of maternal deaths. Proven interventions to prevent maternal mortality are active management of third stage of labour (AMTSL) and the availability of comprehensive emergency obstetric care (CEmOC). Both of these interventions mandate the administration of oxytocin. In Bangladesh there are nonfunctioning institutionalized guidelines from the Director General of Health Services on the storage of oxytocin, which may impact the potency of oxytocin used during labour. To reduce preventable PPH morbidity and mortality, Bangladesh needs to evaluate the potency of current stores of oxytocin used in both in public and private facilities, develop and enforce protocols to ensure the potency of oxytocin, and promote universal access to quality AMSTL and CEmOC services.

\section{Background:}

Declines in the maternal mortality ratio (MMR) have stalled in Bangladesh, with no significant change between 2010 (194/100000 LB) and 2016 (196/1000000 LB) [1]. The MMR has remained static even though access to maternity care services such as antenatal care, institutional delivery and caesarean section have improved considerably during that period of time [1]. Currently $47 \%$ deliveries take place in health facilities: $29 \%$ in for-profit private facilities, $14 \%$ in public facilities, and rest $4 \%$ in NGO facilities. Despite increased access to basic and comprehensive emergency obstetric care services, too many women are still dying around childbirth [2].

\section{Management of Postpartum Hemorrhage:}

Data depicts that majority of maternal deaths take place immediately after birth, and that postpartum hemorrhage (PPH) is the leading cause of MMR. PPH's contribution to maternal mortality, in Bangladesh, has remained unchanged over time, accounting for 31\% of all maternal deaths in both 2010 and 2016. [1]. The 'active management of third stage of labour (AMTSL)' and 'provision of Comprehensive Emergency Obstetric Care (CEmOC) services' are proven interventions to combat PPH related mortality and morbidity [3, 4]. An important component of both CEmOC and AMSTL is administering parenteral oxytocin within one minute after childbirth. The availability and proper use of oxytocin during labor is critical in preventing $\mathrm{PPH}$.

\section{Method:}

We have reviewed BMMS 2016 and BDHS 2017-18 report for cause and rate of maternal mortality estimation[1, 2]. We have also reviewed different government documents[5] and journal articles.

\section{Result:}


A recent study in Bangladesh reported oxytocin administration was suboptimal at $94 \%$ of district hospitals and $71 \%$ of sub-district hospitals [6]. The BMMS 2016 data also shows that only $41 \%$ of public hospitals qualified as functional CEmOC facilities [1].This means that the majority of facilities are not fully compliant with CEMOC and AMSTL protocols. The data also indicate that oxytocin use is suboptimal with issues of availability and, potentially, potency. Unfortunately, in Bangladesh there is no formal guideline on the use and storage of oxytocin in the public and private sector $[7,8]$. The Directorate General of Health Services Bangladesh issued a circular in 2012 for the proper use of oxytocin in health facilities but this has not been institutionalized [5, 9].

\section{Discussion:}

Oxytocin needs to be stored in appropriate temperature controlled conditions and discarded after the manufacturers' "use by" date; otherwise there is a risk that its efficacy will be compromised [10]. In the absence of clear protocols on 'oxytocin storage and use' there is a very real danger that even if administered, as part of AMSTL and CEmOC protocol, it will lack efficacy. If this issue is not addressed, $\mathrm{PPH}$ will continue to kill mothers in Bangladesh, and the national MMR will not improve. We need to ensure that providers correctly follow AMTSL and CEmOC protocols, which include the administration of oxytocin, but we also must ensure the availability and quality of the drug, first. To reduce the maternal mortality due to PPH, the following actions should be prioritized for Bangladesh:

1. Conduct a survey to document the potency of available oxytocin in the public and private sector and take corrective measures to ensure the potency of the oxytocin.

2. Develop and enforce clear guidelines on oxytocin storage for the public and private sector.

3. Ensure all providers, including private hospital providers, are fully compliant with proper implementation of AMSTL protocols.

4. Ensure all facilities (private and public) have CEmOC facilities.

\section{Conclusion:}

Bangladesh has been successful in reducing maternal mortality, however MMRs will remain stubbornly high and mothers will continue to die of preventable PPH deaths if quality oxytocin is not available and used according to AMSTL and CEMOC protocols.

\section{Abbreviations}

AMSTL: Active Management of Third Stage of Labour

BDHS: Bangladesh Health and Demography Survey

BMMS: Bangladesh Maternal Mortality Survey

CEmOC: Comprehensive emergency obstetric care 
MMR: Maternal Mortality Ratio

PPH: Post-Partum Hemorrhage

\section{Declarations}

\section{Policy Recommendation:}

Injection Oxytocin should fit with Child immunization cold chain to continue its potency

\section{Ethics approval and consent to participate}

The used reports are open access and public document. There is no need to take permission. Moreover; data was presented as if no individual can be trace back.

\section{Consent for publication}

Not needed as it is publicly available data and the owner of this data has provided prior consent to publish article with this data

\section{Availability of data and material}

The survey data was already publicly already available

\section{Competing interests}

The author reports no conflicts of interest in this work.

\section{Funding}

This research need no funding. All the authors have contributed voluntary

\section{Authors' contributions}

AR has conceptualized the editorial and led the development of the first draft. AA, TB and IA all have reviewed the editorial and agree to be accountable for all aspects of the work.

\section{Acknowledgements}

Not Applicable

\section{References}

1. National Institute of Population Research and Training, Bangladesh Maternal Mortality and Health Care Survey 2016: Preliminary Report. 2017, National Institute of Population Research and Training Dhaka. 
2. National Institute of Population Research and Training, Bangladesh Demography Health Survey 2017-8: Key indicators. 2019, National Institute of Population Research and Training: Dhaka.

3. Campbell, O.M.R. and W.J. Graham, Strategies for reducing maternal mortality: getting on with what works. The Lancet, 2006. 368(9543): p. 1284-1299.

4. World Health Organization, Monitoring emergency obstetric care. A handbook. 2009, Geneva: World Health Organization.

5. Maternal Neonatla and Child Health, Government order: Storage of oxytocin in public facilitis in Bangladesh. 2012, Maternal Neonatla and Child Health: Dhaka.

6. Billah, S.M., et al., Quality of care during childbirth at public health facilities in Bangladesh: a crosssectional study using WHO/UNICEF 'Every Mother Every Newborn (EMEN)' standards. BMJ Open Quality, 2019.8(3): p. e000596.

7. Directorate Generalof Drug Administration, Bangladesh National Formulary. 4th ed. 2015, Dhaka: Ministryof Health \& Family Welfare, Directorate Generalof Drug Administration.

8. Oliver, V.L., et al., Knowledge, perception and practice towards oxytocin stability and quality: $A$ qualitative study of stakeholders in three resource-limited countries. PLOS ONE, 2018. 13(9): p. e0203810.

9. Hossain, S.M., et al., Maternal health commodity landscaping exercise: A snapshot of the Bangladesh program. 2014.

10. World Health Organization, Stability of Injectable Oxytocics in Tropical Climates: Results of Field Surveys and Simulation Studies on Ergometrine, Methylergometrine and Oxytocin 1993, Geneva: World Health Organization. 50. 